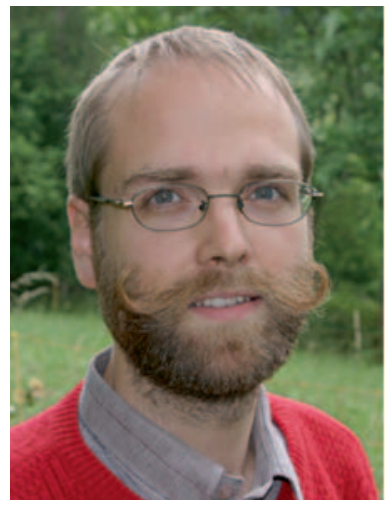

Dr. med. vet. Michael Walkenhorst, Frick, Schweiz

\section{Evidenz und Empirie aus tierärztlicher Perspektive: Ein Blick in die Phytotherapie}

Als Tierarzt wird man oft gefragt, ob die medizinische Arbeit mit Tieren nicht besonders schwierig sei, da doch diese Patienten nicht verbalisieren können, was ihnen fehlt. Einerseits stimmt das; andererseits, und das ist durchaus ein Vorteil, können sie uns auch nicht verbal fehlleiten. Allerdings befasst man sich als Tierarzt kaum mit dem Patienten allein, sondern Diagnostik, Prognostik, Entscheid und Therapie spielen sich gewöhnlich im Spannungsfeld der «Dreiecksbeziehung» Patient - Tierarzt Patientenbesitzer ab.

Anders als die Humanmedizin hat es die Tiermedizin mit erheblich mehr als einer Spezies zu tun, und unsere Patienten sind nicht einmal alles Säugetiere, denn auch Vögel, Reptilien, Amphibien oder Fische können dazugehören. Schon zum «normalen» Praxistag gehören oft mehr als drei Tierarten - bei Zootierärzten regelmässig auch noch ein paar mehr.

Das tierärztliche Entscheidungs-, Verantwortungs- und Tätigkeitsspektrum ist sehr divers und weiträumig. Das Bemühen um das Wohl eines individuellen Tiers kann dabei im Mittelpunkt stehen. Hier weist die Tiermedizin einen ähnlich hohen Spezialisierungsgrad und vergleichbar umfangreiche therapeutische Optionen auf wie die Humanmedizin. Nicht zuletzt sind nahezu alle humanmedizinischen Operations- und Medikationsverfahren zunächst im Tierversuch entwickelt worden. Andererseits ist der Tierarzt in der Nutztierhaltung (als Dienstleister für einen unter den aktuellen Rahmenbedingungen agierenden landwirt- schaftlichen Betrieb) von durchaus nicht unerheblicher ökonomischer Relevanz. Hier ist es zwingend notwendig, die Empfehlung, ein Tier zu behandeln oder zu schlachten, nicht primär aufgrund der medizinischen Prognose, sondern aufgrund einer Kosten-Nutzen-Abwägung auszusprechen. Nicht zuletzt tragen die in der kurativen Praxis, aber auch die in der Lebensmittelhygiene tätigen Tierärzte die Verantwortung für das Wohl des Menschen im Hinblick auf die Qualität und Sicherheit tierischer Lebensmittel.

Die Komplementärmedizin gehört - in den vergangenen 20 Jahren wieder vermehrt - zum therapeutischen Spektrum der tierärztlichen Tätigkeit. In Deutschland hat sich die Gesellschaft für Ganzheitliche Tiermedizin (GGTM e.V.), ein eigenständiger Verein mit mehr als 700 Mitgliedern, diesem Thema verschrieben. In der Schweiz ist die Schweizerische Tierärztliche Vereinigung für Komplementär- und Alternativmedizin (camvet.ch) mit ihren rund 130 Mitgliedern die sechstgrösste der insgesamt 14 Fachsektionen der Gesellschaft Schweizerischer Tierärztinnen und Tierärzte (GST). In der Schweizerischen Medizinischen Gesellschaft für Phytotherapie (SMGP) stellen Tierärzte derzeit rund $8 \%$ der insgesamt gut 700 Mitglieder. In Deutschland und in der Schweiz konnten tierärztliche Weiterbildungstitel beispielsweise zu Homöopathie, Akupunktur und Phytotherapie etabliert werden.

In der Ausgabe der SCHWEIZERISCHEN ZEITSCHRIFT FÜR Ganzheitsmedizin, die Sie heute in den Händen halten, können Sie in eine dieser komplementä(ier)medizinischen Therapieoptionen Einblick nehmen - in die Veterinärphytotherapie. Und dies anhand der Zusammenfassung zum Parallelsymposium Veterinärmedizin, welches anlässlich der Internationalen Tagung «Phytotherapie 2014» im vergangenen Juni in Winterthur auf erfreulich reges Interesse stiess. Das Motto dieses Parallelsymposiums lautete: «Zwischen Empirie und Evidenz - (Re)Aktivierung der Veterinärphytotherapie» [1]. Tatsächlich - und wie vielleicht aus oben Beschriebenem bereits zu erahnen - spielt sich die tägliche tierärztliche Praxis in ganz besonderer Weise im Spannungsfeld zwischen der eigenen Erfahrung und (neuesten) wissenschaftlichen Erkenntnissen ab, wobei die Evidenzebene «Cochrane Review» nicht nur für die tierärztliche Arbeit mit Afrikanischen Elefanten nicht zu erwarten ist ...

So befindet sich im Vergleich zur Humanmedizin der Tiermediziner, unabhängig von der Therapierichtung, die er bevorzugt einsetzt, wesentlich öfter in der Situation, Entscheidungen ohne verfügbare wissenschaftliche Evidenz treffen zu müssen [2]. Die Empirie hat folglich nach wie

\section{KARGER}

(c) 2015 S. Karger GmbH, Freiburg
Dr. med. vet. Michael Walkenhorst 
vor einen besonderen Stellenwert im tierärztlichen Praxisalltag. Jedoch ist die Empirie ja nicht ohne Evidenz - nicht umsonst wird ja die (hoffentlich doch zumindest auf eigenen Erfahrungen basierte) Expertenmeinung als Evidenzstufe IV in der Evidence-Based Medicine durchaus akzeptiert.

Global betrachtet (z.B. in China oder Indien) sind seit Jahrtausenden traditionell basierte Medizinsysteme unterbruchlos bis heute die Basis nicht nur der humanmedizinischen, sondern auch der tierärztlichen Tätigkeit. In Europa ist diese doch zumindest auf Jahrhunderten tierärztlicher Erfahrung beruhende tiermedizinische Therapieform jedoch nahezu erloschen - allerdings noch gar nicht allzu lang, wie sich an Typoskripten veterinärpharmakologischer Vorlesungen aus der Mitte des vergangenen Jahrhunderts ablesen lässt [3].

Aus dem Bedarf, die Empirie systematisch zu dokumentieren, hat sich ein eigenes Forschungsfeld entwickelt, das interdisziplinär sozial- und naturwissenschaftliche Methoden verbindet und zu dem beispielsweise die Ethnobotanik, die Ethnopharmakologie oder auch die Ethnoveterinär- medizin zählen. Mit dieser Methodik wird derzeit in der Schweiz das (noch) vorhandene europäisch-veterinärmedizinische Erfahrungswissen, insbesondere die Behandlung von Nutztieren mit Arzneipflanzen, erfasst: Bäuerinnen und Bauern haben diese Tradition in der Schweiz bis heute erhalten. Dank ihrer grosszügigen Kooperationsbereitschaft konnten in den vergangenen Jahren verschiedene ethnoveterinärmedizinische Forschungsprojekte erfolgreich abgeschlossen werden [4], und weitere laufen aktuell oder sind in Planung.

Mit dem Parallelsymposium Veterinärmedizin anlässlich der Tagung «Phytotherapie 2014» wollten die Organisatoren aktiv anregen, das reichhaltige Potenzial der Arzneipflanzen in der veterinärmedizinischen Forschung, Lehre und Praxis wieder verstärkt zu nutzen. Ob und inwieweit das gelungen ist, muss die Zukunft zeigen. Diese aktiv mitzugestalten ist eine spannende Aufgabe für uns alle. Ich wünsche Ihnen hierbei und natürlich insbesondere beim Lesen der aktuellen Ausgabe der SCHWEIZERISCHEN ZEITSCHRIFT FÜr GANZHEITSMEdIZIN viel Freude.

\section{Literatur}

1 Walkenhorst M, Vogl CR, Vogl-Lukasser B, Vollstedt S, Brendieck-Worm C, Ivemeyer S, Klarer F, Meier B, Schmid K, Disler M, Bischoff T, Hamburger M, Häsler S, Stöger E: Zwischen Empirie und Evidenz - (Re)Aktivierung der Veterinärphytotherapie. Forsch Komplementmed 2014;21(suppl 1);35-42.
2 Cockcroft PD, Holmes MA (eds): Handbook of Evidence-Based Veterinary Medicine. Oxford/Malden/Carlton, Blackwell, 2003, pp 2-7.

3 Steck W: Pharmakologie. Typoskript, erstellt von Gross R. Bern, 1944. (Kopie im Archiv der Schweizerischen Vereinigung für Geschichte der Veterinärmedizin.)
4 Schmid K, Ivemeyer S, Vogl CR, Klarer F, Meier B, Hamburger M, Walkenhorst M: Traditional use of herbal remedies in livestock by farmers in 3 Swiss cantons (Aargau, Zurich, Schaffhausen). Forsch Komplementmed 2012; 19:125-136. 